\title{
Pertinencia ocupacional de los administradores financieros de la Corporación Universitaria Minuto de Dios (vicerrectoría Bogotá sur) desde el año 2015
}
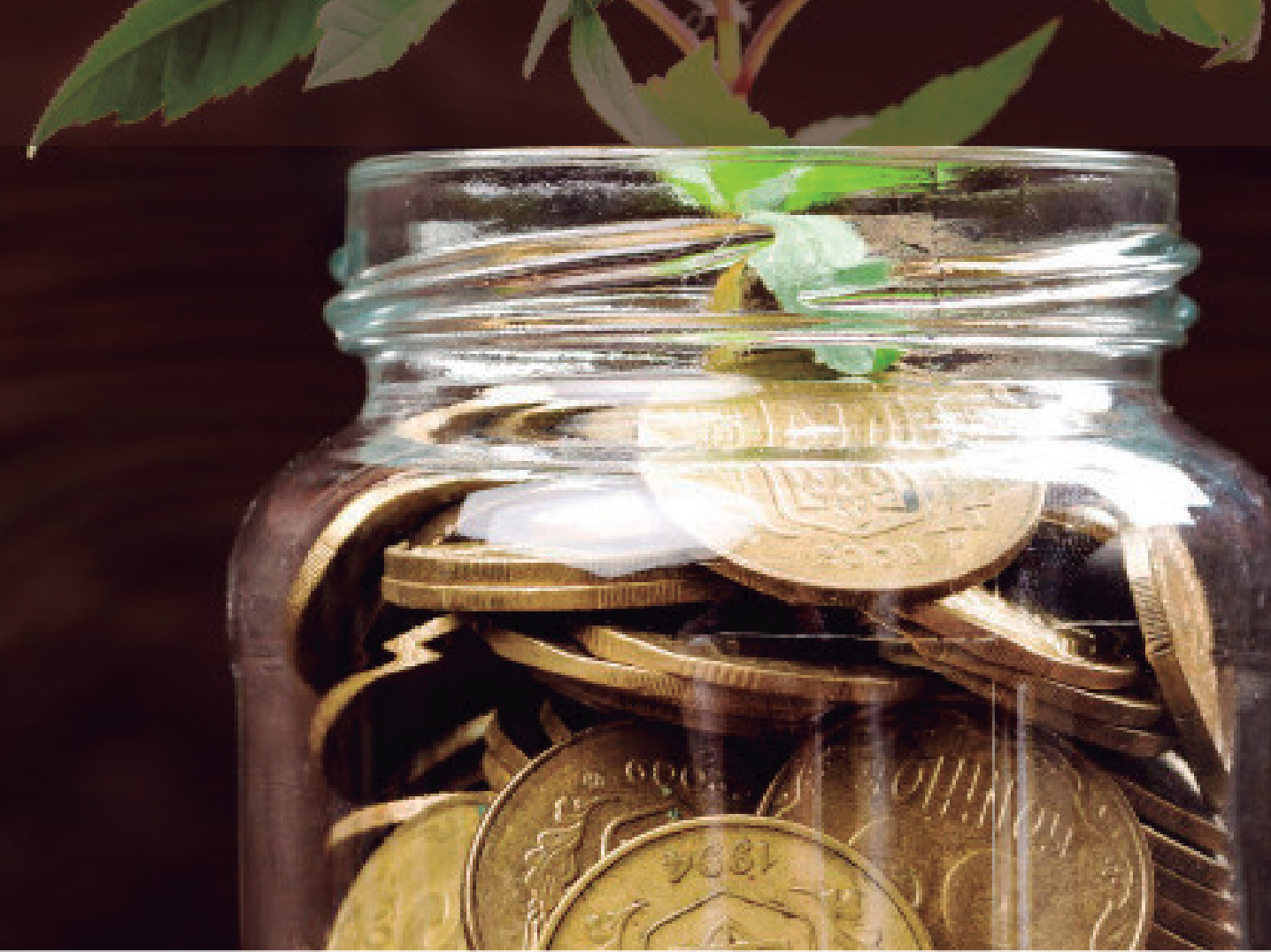

CLAUDIA LILIANA SERRANO JUNCO ID https://orcid.org/0000-0001-6844-2735 Corporación Universitaria Minuto de Dios / claudia.serrano@uniminuto.edu.co

DIANA GERALDINE MATEUS DURAN (10) https://orcid.org/0000-0002-2543-2729 Corporación Universitaria Minuto de Dios / dmateusdura@uniminuto.edu.co

MARY LEIDY BERMÚDEZ GÓMEZ https://orcid.org/0000-0001-8296-8549 Corporación Universitaria Minuto de Dios / mbermudezg2@uniminuto.edu.co

MIGUEL ANDRÉS BERNAL GONZÁLEZ https://orcid.org/0000-0001-7572-5119 Corporación Universitaria Minuto de Dios / mbernalgon1@uniminuto.edu.co

LUZ ADRIANA PINZÓN PEDRAZA ID https://orcid.org/0000-0003-4878-0280 Corporación Universitaria Minuto de Dios / Ipinzonp@uniminuto.edu.co 
Artículo de investigación

Recepción: 03 de febrero de 2020

Aceptación: 28 de julio de 2020

Cómo citar este artículo

Serrano Junco, C., Mateus Durán, D., Bermúdez Gómez, M., Bernal González, M., y Pinzón Pedraza, L. (2020). Pertinencia ocupacional de los administradores financieros de la Corporación Universitaria Minuto de Dios (vicerrectoría Bogotá sur) desde el año 2015. Revista Colombiana de Ciencias Administrativas, 2(1), pp. 8 - 25 Recuperado a partir de:

http://cipres.sanmateo.edu.co/index.php/rcca

\section{Pertinencia ocupacional de los administradores financieros de la Corporación Universitaria Minuto de Dios (vicerrectoría Bogotá sur) desde el año 2015}

\section{Resumen:}

Para las Instituciones de Educación Superior (IES) es importante realizar seguimiento a los graduados con el fin de garantizar el desarrollo progresivo de educación hacia estándares de alta calidad. Adicional, según lo suscrito en los Decretos 2566 del 10 de septiembre de 2003 y el 1001 del 03 de abril de 2006, enmarcados bajo la Ley 30 de 1992; es necesario contar con un acompañamiento y seguimiento a todos sus graduados; además, es deber de las instituciones graduar estudiantes que sean pertinentes en el mercado laboral. Por tal razón, en el presente artículo, se exponen los resultados de una investigación con enfoque mixto que permite medir las variables de un grupo determinado; para ello se usaron métodos estadísticos, y a partir de este análisis, se establecieron conclusiones respecto a las hipótesis planteadas. Los resultados obtenidos permitieron diagnosticar la pertinencia ocupacional de los graduados del programa de administración financiera de la Corporación Universitaria Minuto de Dios (Uniminuto) vicerrectoría Bogotá sur desde el año 2015.

Palabras clave: empleabilidad; egresados; universidades; pertinencia; mercado laboral. 


\section{Financial Administrators' occupational relevance from Corporation Universitaria Minuto de Dios (south Bogotá vice-rectory) since 2015}

\section{Abstract:}

For the Superior Education is important to monitor graduated students in order to guarantee the progressive development of education toward high quality standards. In addition, according to the orders 2566 from September 10, 2003 and 1001 from April 03, 2006, related to the law 30 (1992); it is necessary to have an accompaniment and follow-up to all its graduated students; also, the institutions have the duty to graduate relevant students in the labour economics. For this reason, in the present paper is exposed the research with a mix focus. It allows the variables measure of a determined group. In this order, it is used statistica methods, and it stablishes conclusions from this analysis regarding the hypotheses proposed. The results obtained will allow us to diagnose the occupational relevance of the graduated students of the Financial Administration Program from Corporación Universitaria Minuto de Dios (Uniminuto) since 2015.

Keywords: employability; graduate; universities; relevance; working market.

\section{Introducción}

La formación académica y el mercado laboral han ido estrechando su vínculo e interdependencia con el pasar de los tiempos. Como resultado, generan un impacto determinante en el perfil profesional de los graduados de las diferentes disciplinas académicas (Jaramillo, 2015). En la actualidad, el mercado laboral determina los factores relevantes que debe cumplir un profesional para acceder a una plaza de empleo. Por lo anterior, el Gobierno colombiano, en cabeza del Ministerio de Educación (MEN), ha creado el Observatorio Laboral para la Educación (OLE) en aras de garantizar que las ofertas educativas y la realidad laboral sean pertinentes. Permite monitorear de forma constante la relación entre la preparación académica universitaria; así como el desempeño de los graduados al momento de ingresar al mercado laboral. Así, la pertinencia se convierte en un factor prioritario al momento de evaluar a las instituciones que ofertan programas de educación superior (OLE, 2005). 
La Corporación Universitaria Minuto de Dios (Uniminuto) entiende que los programas de alta calidad son reconocidos mediante el desempeño laboral de sus graduados y el impacto que tienen en el desarrollo social, económico y cultural de su entorno (Uniminuto, 2018). Por consiguiente, dentro de los factores de evaluación del programa, se tiene un apartado especialmente dedicado a medir el seguimiento y cumplimiento de los indicadores relacionados con el impacto de los egresados en el medio. Desde allí se evalúan aspectos tales como la existencia de registros actualizados sobre ocupación y ubicación profesional de los egresados del programa; correspondencia entre la ocupación y ubicación profesional; apreciaciones de egresados, empleadores y agentes externos. Además, utilización óptima de la información contenida en el OLE; análisis de los procesos que involucran la evaluación de la situación actual de los egresados; mecanismo y estrategias que surjan y las cuales conlleven a realizar ajustes al programa en atención a las necesidades del entorno. Por último, estrategias para afrontar el mundo laboral; así como índices de empleabilidad; egresados que forman parte activa de grupos comunitarios, culturales, entre otros.

En este sentido, la presente investigación surge como tema de conformación para un semillero de investigación de la Uniminuto(vicerrectoría Bogotá sur) que contribuya a mejorar la evaluación del programa en cuanto a los temas relacionados con la pertinencia ocupacional de los graduados de administración financiera, en lo que concierne a los procesos educativos que adelantaron en la Institución. Esta investigación es apropiada y enriquecedora para graduados, estudiantes y directivos de la universidad; es imperativo determinar si el programa que ofrece la institución educativa está acorde y guarda directa relación con la realidad laboral que viven los administradores financieros de Uniminuto en la actualidad.

\section{Impacto y retos de los programas educativos de las IES. Una aproximación a la conceptualización de la Uniminuto.}

El impacto de los programas educativos en el desarrollo económico del país trae consigo retos y ajustes inmediatos en la heterogeneidad de programas que ofrecen las IES colombianas (Parra, Vergel y Sánchez, 2015). En este sentido es importante que las universidades creen nuevos procedimientos y modelos educativos que les permitan dinamizar la oferta educativa y estrechar la pertinencia de la misma con el mercado laboral (Parra, Vergel y Sánchez, 2015). Sin embargo, como lo explica el gerente general para Colombia (experta en gestión del talento humano), de la compañía Lee Hecht Harrison, los profesionales en la actualidad deben tener la capacidad de anticiparse a los giros que puede traer la globalización 
y el impacto que estos tendrán en los cargos que desempeñan (Portafolio, 2018). No obstante, se cuestiona si las IES realmente están preparando a los futuros graduados para estos retos; aún más, si de acuerdo con el programa sus contenidos académicos guardan directa relación con lo esperado por el mercado laboral dinámico y constante en el que vivimos (Portafolio, 2018).

Es importante tener en cuenta la realidad de los egresados de las universidades al momento de incorporarse en el mercado laboral, considerando que no siempre el éxito está garantizado y no todos los estudiantes se adecuan a las exigencias de las empresas (Universia, 2014). A partir de estas limitaciones los currículos universitarios cobran importancia en la preparación del graduado y en la inserción del mismo en el mercado laboral. Las compañías son cada vez más exigentes en su perfilamiento laboral haciendo que cada individuo que oferta su servicio tenga que estar más acorde al sistema (Escobar, 2005).

La Uniminuto inició a sus labores en el año 1992 con 240 estudiantes en Bogotá; buscaba el desarrollo humano integral mediante el acceso a la educación, el trabajo y el arte (Uniminuto, 2017). En el año 2007, alienados al Plan de Educación Nacional y Distrital "Acceso y permanencia a la educación para todas y todos", la Uniminuto firma un acuerdo con la Secretaria de Educación del Distrito que dio vida a Uniminuto Vicerrectoría Regional Bogotá Sur (VBS). Su propósito fue brindar las oportunidades de acceso a la educación tecnológica y profesional a los jóvenes de las localidades del sur de la ciudad (VBS, 2015) que les permitirá un desarrollo social y económico de forma individual y colectiva. El programa de administración financiera hace parte de la facultad de ciencias empresariales y se empezó a ofertar en la Uniminuto VBS desde el año 2011 (VBS, 2015).

El programa de administración financiera se imparte en modalidad presencial y a distancia. Su principal objetivo es lograr en sus egresados competencias en la gestión y aprovechamiento de los recursos financieros que permitan el desarrollo social y económico de la comunidad en la cual se vaya a desempeñar (Uniminuto, s.f.). El profesional graduado de Uniminuto VBS es formado bajo tres (3) áreas generales: el desarrollo humano, competencias profesionales y responsabilidad social. Lo anterior a fin de estar alineados con los pilares de la universidad y su interés en la transformación positiva y exponencial de la sociedad (Financiera, 2013). 
Es así como un profesional graduado de administración financiera de Uniminuto VBS será reconocido por conocerse a sí mismo y tener la capacidad de mantener relaciones sanas con las personas de su entorno. Asimismo, tendrá todas las competencias académicas, metodológicas, analíticas, administrativas e innovadoras para guiar y transformar financieramente a empresas, socios, accionistas y personas en general. Por último, sobresale por su capacidad de reconocer realidades de su entorno y llevar sus acciones al mejoramiento de las condiciones de una comunidad mediante el desarrollo social y económico de la sociedad (Financiera, 2013).

En busca del mejoramiento continuo de los programas académicos que oferta la universidad, dentro de las políticas de evaluación y seguimiento se establecen balances anuales a los diferentes programas. En la evaluación realizada al programa de administración financiera de la Uniminuto VBS (2018), de forma general, en el Factor 9 (Impacto de los egresados en el medio) se concluyó que para mejorar el nivel de calificación obtenido es necesario hacer un seguimiento constante por parte del programa a los graduados. Lo anterior, a fin de determinar la apreciación que tienen sobre las competencias generales y específicas para el desarrollo laboral. Además, desde la Uniminuto VBS conocer de forma real el mercado laboral en el cual se desempeñan los graduados de administración financiera y la relación que este guarda con la formación académica impartida por la IES. A partir de lo anterior, generar procesos de evaluación de los contenidos del programa y crear estrategias de innovación que les permita formar profesionales competentes y preparados de forma consecuente a las necesidades del talento humano que demanda el mercado actual (Uniminuto, 2018).

Sin embargo, es necesario entender esta evaluación a profundidad; en su estructura guarda detalles que hacen pertinente la presente investigación y soportan el problema a trabajar en la misma. Al analizar a fondo los datos, se encuentra que en el Factor 9 (impacto de los egresados en el medio), característica 36 (seguimiento a egresados), punto b, es decir, la correspondencia entre la ocupación y ubicación profesional de los egresados y el perfil de formación del programa.

La meta para el indicador es "poseer un análisis debidamente documentado que refleje el nivel de correspondencia entre la ocupación y la ubicación profesional de los graduados y el perfil de formación del programa, por lo menos una vez al año" (Uniminuto, 2018). Se obtuvo un puntaje de 30 en la calificación específica (en la escala de 0 a 100), ubicándose en la categoría "No se cumple"; significa que existe un documento pero no se evidencia todo lo solicitado y no es oficial. Al revisar la ficha técnica 
del aspecto, se encuentra que es deber de la universidad (en cabeza de la coordinación de egresados) realizar cada año un análisis de la pertinencia ocupacional de sus graduados. Sin embargo, en el registro de seguimiento se evidencia que en el año 2015 fue la última vez que se realizó esta labor.

Luego del análisis de la evaluación y sus resultados, se concluye que existe un problema directamente relacionado con el programa de administración financiera. A la fecha no se está cumpliendo de forma total con lo exigido en el contenido de la evaluación anual del programa, en lo que respecta al aspecto b: Correspondencia entre la ocupación y ubicación profesional de los egresados y el perfil de formación del programa. Por lo anterior, se formula la siguiente pregunta de investigación, a partir de la cual se obtendrán resultados que generarán valor al programa y permitirá mejorar la calificación de cumplimiento del aspecto b, para la posterior evaluación de procedimiento que se efectué.

¿Es pertinente la ocupación laboral de los administradores financieros de Uniminuto VBS?

\section{¿Por qué la importancia de estudiar la pertinencia ocupacional?}

Los procesos educativos deben estar en constante transformación por cuanto las formas de empleabilidad varían proporcionalmente de acuerdo con las necesidades socioeconómicas de la población y los constantes cambios en el mercado económico nacional. Por tanto, el Plan Nacional Decenal de Educación (PNDE) 2006-2016 establece que:

En reconocimiento constitucional como un país multicultural, pluriétnico, diverso y biodiverso, la educación es un derecho cumplido para toda la población y un bien público de calidad, garantizado en condiciones de equidad e inclusión social por el Estado, con la participación co-responsable de la sociedad y la familia en el sistema educativo. La educación es un proceso de formación integral, pertinente y articulado con los contextos local, regional, nacional e internacional que desde la cultura, los saberes, la investigación, la ciencia, la tecnología y la producción, contribuye al justo desarrollo humano, sostenible y solidario, con el fin de mejorar la calidad de vida de los colombianos y la superación de la pobreza y la exclusión (MEN, 2006). 
Como bien lo cita el PNDE, la educación debe ser integral, pertinente y articulada al contexto regional, nacional e internacional (Educación, 2014). Sin embargo, para que esto se logre de manera efectiva, se deben determinar las diferentes formas de empleabilidad de las que son participes los graduados en la actualidad. Lo anterior, a fin de entender las necesidades del mercado laboral y a partir de esto estructurar planes de estudio que satisfagan las expectativas de talento humano de empresarios y emprendedores del país (Emiliani, 2015).

Investigar la pertinencia en el ámbito social de los graduados de la carrera de administración financiera de la Uniminuto, se hace importante pues es una manera de identificar el impacto real que está generando el profesional saliente de esta universidad en su entorno social. Así, permite que pueda aprovechar las oportunidades de mejora, que se evidencien en pro del desarrollo progresivo de la calidad de la educación. De esta manera, cumple con el compromiso como institución de garantizar programas académicos que permitan la inclusión efectiva dentro de las oportunidades de empleo y emprendimientos que la sociedad pueda ofrecer (Vives, 2013).

Por otra parte, bajo el marco legal colombiano se hace justificable establecer estudios que evalúen la pertinencia de los graduados de las universidades. Según lo dicta la Ley 30 de 1992, los egresados son un elemento importante dentro del mejoramiento de la calidad de la educación; es imperativo realizar seguimiento a los mismos. Ahora bien, de acuerdo con el Decreto 2566 de septiembre 10 de 2003 y el Decreto 1001 de abril 03 de 2006, las instituciones de educación superior tienen como obligación demostrar políticas y estrategias que permitan verificar el desempeño laboral, el aprovechamiento de los procesos académicos, el intercambio de conocimiento con otros egresados e impacto que causan sus graduados en el ámbito social. Esto, con el fin último de reestructurar cualquier vacío que se evidencie en estos procedimientos; así como garantizar un mejoramiento continuo de la educación en instituciones pertenecientes a las IES.

\section{Conceptos referenciados en la pertinencia ocupacional.}

\section{Ministerio de Educación Nacional (MEN).}

Es una entidad de orden nacional cuyo objetivo es trabajar de forma constante e ininterrumpida para que los colombianos tengan una educación de calidad, competitiva y pertinente. En este sentido, que permita disminuir las brechas sociales, políticas y educativas que se presentan en nuestro país (MEN, 2006). 


\section{Sistemas de Información del Ministerio de Educación Nacional (SNIES).}

Sistema que recopila y organiza la información relevante sobre la educación superior. Permite hacer planeación, monitoreo, evaluación, asesoría, inspección y vigilancia del sector.

\section{Observatorio Laboral para la Educación (OLE).}

Es una herramienta web que permite analizar la pertinencia de la educación a partir del seguimiento a los graduados y su empleabilidad en el mercado laboral.

\section{Instituciones de Educación Superior (IES).}

Aquellas instituciones reconocidas oficialmente como prestadoras del servicio de educación superior en Colombia (MEN, 2011). Las IES se clasifican en A, según su carácter académico e identidad del campo de acción; en lo académico le permite ofertar y desarrollar en programas de educación superior. También se clasifican en B según su naturaleza jurídica ya sea pública o privada (MEN, 2010).

\section{Graduado y egresado.}

De acuerdo con la definición establecida por el MEN, un graduado es aquel que ha recibido un título de grado por parte de una IES luego de la culminación de sus estudios. Un egresado es aquel que ha terminado sus materias, pero no ha obtenido un título de grado por parte de una IES (MEN, 2011).

\section{Procesos de enseñanza y aprendizaje.}

Las IES tienen como reto formar profesionales que cumplan con las exigencias del mercado laboral, sin embargo, los procesos de enseñanza y aprendizaje son de vital importancia para lograr este cometido. Este tipo de procesos son concebidos como un espacio en el cual el estudiante y el profesor son figuras protagónicas; uno desempeña el rol de constructor del conocimiento (estudiante) y el otro como facilitador de los procesos de aprendizaje (profesor) (Marista, s.f.). Bajo este modelo se construyen planes de estudio que tienen como fin abarcar todas las áreas de formación que los profesionales necesitan para enfrentar los retos de empleabilidad. En 
un panorama ideal, la sinergia entre graduados e institución debe permitir un enriquecimiento mutuo, partiendo de la formación académica impartida por la institución y las experiencias de la vida laboral de los graduados que dan valor a las estructuras educativas de las instituciones (Educación, 2016).

\section{Mercado laboral.}

Es el escenario en donde confluyen la oferta y demanda de trabajo convirtiéndose así en uno de los principales aspectos que afectan la economía de un país (Ustabuca, s.f.). En este escenario se pueden analizar las variaciones de las tasas de empleo, las actividades o labores en las que se desempeñan las personas, las necesidades de mano de obra requeridas por las empresas, entre otros. Al ser un ecosistema influyente en la economía, es de naturaleza cambiante; factores como la globalización o la implementación de las nuevas tecnologías impactan de forma directa; generan grandes cambios y afectan a sus principales protagonistas, empleados y empleadores.

\section{Empleabilidad.}

De acuerdo con la teoría del capital humano, lo que marca la diferencia entre individuos en el mercado laboral es la calificación formal que posee el trabajador (Formichella, 2011). En pocas palabras, de acuerdo con esta teoría la educación (formal y no formal) resulta ser un aspecto diferenciador importante en el mercado laboral. Sin embargo, es común encontrar en el mercado laboral dos (2) individuos con el mismo nivel de educación pero que por características diferentes a la formación son desiguales el uno del otro.

A lo anterior se le llama empleabilidad, consiste en la "habilidad que tiene una persona de conseguir o mantener un empleo" (Formichella y London, 2013). Entonces, el concepto de empleabilidad es entendido como las características de las cuales está dotado un individuo y lo llevan a resaltar por encima de otros. Estos atributos abarcan:

Las calificaciones, los conocimientos y las competencias que aumentan la capacidad de los trabajadores para conseguir y conservar un empleo, mejorar su trabajo y adaptarse al cambio, elegir otro empleo cuando lo deseen o pierdan el que tenían e integrarse más fácilmente en el mercado de trabajo en diferentes períodos de su vida. (Weinberg, 2004) 


\section{Contextualización teórica en el marco de la pertinencia ocupacional.}

El fin último de todo proceso de enseñanza de aprendizaje es la formación de ciudadanos competentes para un mundo globalizado, con la capacidad de transformar realidades sociales para el beneficio general de la comunidad (MEN, 2009). En dicho objetivo, las IES como entidades de formación de profesionales, tecnólogos y técnicos, se encuentran en la necesidad de llevar a cabo un proceso de reclutamiento de estudiantes; así como formación académica de los mismos en las diferentes especialidades de la oferta de la institución y, por último, un seguimiento de la condición laboral o las actividades que se encuentran ejerciendo los graduados del plantel (Congreso de Colombia, 1992). Sin embargo, es importante reconocer qué espera el mundo laboral colombiano de los administradores financieros. Aún más importante, determinar si la formación académica impartida por las universidades, a los futuros graduados, es suficiente para satisfacer la demanda laboral.

En 1998 el Señor Omar Cedeño de la Universidad del Valle desarrolló una investigación que pretendía definir el rol del administrador financiero a finales del siglo XX. En esta se concluyó que tiene un papel determinante en dos (2) grandes áreas de influencia de una compañía: la gestión de fondos y el control financiero (Cedeño, 1998). Por tanto, esto nos lleva a vislumbrar que un profesional de la administración financiera puede ser transversal en cualquier compañía que requiera un decisor encargado de tomar la vocería en temas financieros, de inversión, estructura, pagos, seguros, riesgos, dividendos, etc. Además, un auditor que se encargue de velar por la contabilidad financiera, costos, impuestos, análisis y presupuestos (Cedeño, 1998).

El grupo de investigación en Dirección de Empresas (GIDE), de la Fundación Universitaria Esumer ubicada en Medellín, Colombia, en el año 2011 desarrolló el proyecto de investigación "Rediseño Curricular del Programa de Administración Financiera". Lo anterior, con el fin de aportar nuevo conocimiento que sirviera de base para la estructuración de un contenido académico que permita darle administradores financieros al mercado laboral con capacidades alineadas con los referentes teóricos, laboral, las perspectivas de crecimiento económico, el mercado de capitales y las tendencias en finanzas (Vélez, 2011). En este estudio se concluyó que el administrador financiero debe ser formado bajo un sistema de aprendizaje que lo lleve a desarrollar conocimientos, habilidades y valores que impacten 
su entorno. Asimismo, se debe garantizar que el profesional posea conocimiento profundo en áreas de la economía, contabilidad, gerencia, mercadeo, investigación, sistemas de información e idiomas (Vélez, 2011).

De acuerdo con el informe de tendencias laborales realizado por el portal web elempleo.com, para el último trimestre de 2018 la administración financiera se posicionó como la quinta profesión con 4.322 ofertas laborales en Colombia. En este sentido, se determinó que los administradores financieros se ubican en la sexta posición entre los profesionales que más buscan trabajo en esta plataforma (El Empleo, 2019). Sin embargo, al ahondar en estas cifras, tanto la oferta como la demanda de empleo se encasilla en cargos tales como gerente financiero, director administrativo y financiero, contralor, tesorero, auditor, analista contable, analista financiero, entre otros (El Empleo, 2019).

Lo anterior nos lleva a pensar que el rol del administrador financiero sigue como en 1998 en donde se consideraba que sus funciones debían estar enmarcadas en el gasto y la vigilancia, desconociendo por completo que un profesional de la administración financiera debe participar activamente en los procesos de la empresa. Además, participar en negociaciones, mercadeo, inversión y apoyar las áreas de recursos humanos, producción, comercialización, ventas, administrativas, entre otras (Garzón, 2017). Su objetivo consiste en mantener a todas las áreas en una correlación financiera de la cual se puedan tomar datos pertinentes que permitan la toma de decisiones desde la realidad actual y aterrizada de la organización; a fin de cumplir el mayor y más grande objetivo de un administrador financiero: la maximización del valor.

Unavez consideradala posición privilegiada que ocupalaadministración financiera en el estudio de tendencias publicado por el empleo.com, se podría concluir apresuradamente que la pertinencia entre profesión y empleo es un tema de interés para todos los entes relacionados con este tema, en especial las universidades. Sin embargo, luego de realizar una investigación bibliográfica encontramos que hay carencia de este tipo de estudios que permitan determinar los cargos específicos en los cuales se están empleando los administradores financieros; nuevas formas de empleo que han surgido en el mercado laboral y la relación entre lo aprendido en la academia y lo desempeñado en la vida laboral. Universidades como la EAN, Uniminuto, Cooperativa de Colombia, Universidad de Cundinamarca, Universidad del Valle, Universidad de Medellín, entre otras (Moreno y Mendez, 2018) han publicado estudios relacionados a este tema. No obstante, siempre con la 
lupa puesta en el perfil ideal del administrador financiero de acuerdo con los cambios constantes en la economía, sociedad y política, pero dejando de lado la realidad actual de los graduados en cuanto a las formas como se están empleando.

\section{Abordaje metodológico para la visibilización de la pertinencia ocupacional.}

En el libro Metodología de la investigación de Roberto Hernández Sampieri, Carlos Fernández Collado y Pilar Baptista Lucio, se define la investigación como un conjunto de procesos sistemáticos y empíricos que se utilizan para el estudio de un fenómeno especifico, resaltando para ello tres (3) enfoques: Cuantitativo, cualitativo y/o mixto. presente investigación utiliza un enfoque mixto que nos permite medir las variables de un grupo determinado, utilizando métodos estadísticos y estableciendo a partir de este análisis conclusiones respecto a las hipótesis planteadas. Para ello se aplicó una encuesta a una población de 300 graduados de administración financiera de Uniminuto VBS; además, se realizó una revisión bibliográfica que permitiera enriquecer de manera cualitativa la investigación, de tal forma que explique los fenómenos a identificar en el análisis de los datos obtenidos de la encuesta.

En este sentido, se realizó un estudio bibliográfico a las 20 mejores universidades del país, agrupando los hallazgos en cuatro (4) grandes categorías: Registro de egresados y graduados; pertinencia ocupacional; empleabilidad e impacto de los egresados en la estructura académica. Lo anterior permitió consolidar información similar y compararla con la realidad de la Uniminuto a fin de determinar las oportunidades de mejora o potencializar actividades propias que han sido beneficiosas para el programa.

Por otra parte, al interior de la universidad se hizo una revisión documental en cuanto a la información relacionada con los egresados y su pertinencia ocupacional. Así, se logró determinar el último año en el cual se realizó un análisis de pertinencia ocupacional; además, se identificó el estado actual de este proceso al interior de la universidad. A su vez, se aplicó una herramienta de recolección de información a más de 300 graduados del programa de administración financiera de la Uniminuto VBS. Por último, producto del análisis y posterior estudio, se generaron resultados que permitieron evidenciar las características del estado actual de los graduados, así como su pertinencia ocupacional. 


\section{Resultados y conclusiones}

De acuerdo con los métodos aplicados se logró determinar que las universidades entienden que la calidad de la educación que imparten se ve reflejada en el desempeño laboral de sus graduados y el impacto de estos sobre el mercado laboral. Asimismo, se evidencia que los graduados hacen parte de la estructura de la cadena de transformación social, económica y política país, en la medida que su capacidad productiva está reflejada en lograr desempeñarse en el campo de acción según la formación disciplinar, en este caso, administración financiera.

Por otra parte, al interior de la Uniminuto VBS en su evaluación anual se resalta al programa de administración financiera el reconocimiento a los egresados como actores fundamentales en el cumplimento de los retos que trae consigo la MEGA Uniminuto para los años posteriores. En este sentido, el programa requiere fortalecer las estrategias y herramientas que garanticen la empleabilidad y el empleo de sus graduados en el mercado laboral, en la medida que se pierde el seguimiento de los graduandos luego de finalizar su proceso académico.

Respecto a los resultados de la encuesta, se logra concluir que luego de graduarse como profesional el $65 \%$ no obtuvo ascenso o reconocimiento alguno por este logro. Asimismo, solo el 26\% trabaja en el sector financiero, desempeñándose en cargos como analista o auxiliar (38\%). Esto encamina hacia el objetivo general, es importante considerar que la administración financiera es una carrera transversal a los sectores económicos, sin embargo, se hace necesario considerar si para una completa pertinencia los graduados de pregrado requieren estudios superiores de posgrado que les permita fortalecer sus competencias y crecer de forma estable en el ámbito laboral.

Finalmente, se determina que la profesión de administración financiera es un área de estudio subyacente a otras áreas disciplinares que generalmente se confunde con la administración de empresas, la economía - la contabilidad. Claramente, aunque se tocan áreas dentro de los currículos afines a la economía, el enfoque está direccionado a las finanzas en el contexto. Por ello es imperante que los planes curriculares apunten a estas dinámicas académicas al interior de las IES. 


\section{Referencias}

Cedeño, O. (1998). El rol del administrador financiero a finales del siglo XX. Cuadernos de administración, 16(24), pp. 95-104.

Congreso de Colombia. (1992). Ley 30 de diciembre 28 de 1992. Bogotá.

Educación, O. L. (2014). Ministerio de Educación Nacional. Obtenido de http://bi.mineducacion.gov.co:8380/eportal/web/men-observatoriolaboral/ubicacion-geografica.

El empleo. (2019). Informe de tendencias [en línea]. Recuperado de http://elempleo.com

Emiliani, Á. (2015). La pertinencia de la educación para el trabajo. La Silla Vacia. Recuperado de: http://lasillavacia.com/silla-llena/red-de-la-educaci-n/ historia/la-educaci-n-para-el-trabajo-y-la-necesidad-de-su.

Escobar, M. (2005). Las competencias laborales: ¿La estrategia laboral para la competitividad de las organizaciones? Estudios Gerenciales, 21(96), pp. $31-55$.

Formichella, M., y London Silvia. (2013). Empleabilidad, educación y equidad social. Revista de Estudios Sociales 47, pp. 79-97.

Garzón Torres, V. (2017). Tendencias y líneas de desarrollo de la profesión de Administración financiera a nivel regional y nacional. (Tesis de pregrado). Corporación Universitaria Minuto de Dios, Bogotá, Colombia.

Hernández Sampieri, R., Fernández Collado, C. y Baptista, M. (2014). Metodología de la Investigación. Ciudad de México: Mcgraw-Hill.

Jaramillo, O. (2015). Pertinencia del perfil de los profesionales de la información con las demandas del mercado laboral. Revista Interam, 18(2), pp. 111-120.

Las ventajas de pertenecer a una asociación de egresados. (agosto, 2017). Semana. Recuperado de http://www.semana.com/ 
Los retos laborales: El secreto está en anticipar los cambios. (junio, 2018) Portafolio. Recuperado de: http://www.portafolio.co/negocios/retoslaborales-el-secreto-esta-en-anticipar-los-cambios-517708

Marista. (s.f.). Proceso de enseñanza-aprendizaje. Universidad Marista de Mérida. Recuperado de: http://www.marista.edu.mx/p/6/proceso-deensenanza-aprendizaje

MEN. (2006). Decreto 1001 de Abril 3 de 2006. Ministerio de Educación Nacional, Bogotá.

MEN. (2009). Altablero: El periódico de un país que educa y que se educa. Ministerio de Educación Nacional. Recuperado de: https://www. mineducacion.gov.co/1621/article-183911.html.

MEN. (2010). Instituciones de Educación Superior (IES). Ministerio de Educación Nacional . Recuperado de: https://www.mineducacion.gov. co/1621/article-217744.html.

MEN. (2011). Glosario. Ministerio de Educación Nacional. Recuperado de: https://www.mineducacion.gov.co/sistemasdeinformacion/1735/w3article-254707.html.

Moreno, N., y Mendez, A. (2018). Estudio de pertinencia del programa administración de empresas de la Universidad de Cundinamarca seccional Girardot en la comunidad estudiantil 2017. (Tesis de pregrado). Universidad de Cundinamarca, Girardot, Colombia.

OLE. (2005). Observatorio Laboral para la Educación. Ministerio de Educación Nacional. Recuperado de: https://www.mineducacion.gov. co/1621/article-136421.html.

Parra, H., Vergel, M. y Sánchez, J. (2015). Hacia un modelo para evaluar la pertinencia social en la oferta académica de la Universidad Francisco de Paula Santander (Propuesta de trabajo). Ministerio de Educación Nacional. Recuperado de: https://www.mineducacion.gov.co/1759/articles-341895_ archivo_pdf.pdf.

Uniminuto. (s.f). Programas académicos [en línea]. Recuperado de http://www.uniminuto.edu/web/bogotasur/programas-academicos/-/ programa/Rafael+Uribe+Uribe/administracion-financie-2 
Uniminuto. (2018). Factor 9 Impacto de los Egresados en el Medio. Bogotá.

Uniminuto, P. (2017). Historia. Uniminuto. Recuperado de: http://www. uniminuto.edu/historia.

Universia. (2014). ¿Cuáles son las exigencias del mercado laboral? Universia Colombia. Recuperado de: http://noticias.universia.net.co/enportada/noticia/2014/03/28/1092103/cuales-son-exigencias-mercadolaboral.html.

Ustabuca. (s.f.). Mercado laboral. Universidad Santo Tomas. Recuperado de:http://www.ustabuca.edu.co/gpresenzia/vista/tpl/ustabmanga/mercadolaboral.html.

VBS. (2015). Historia VBS. Uniminuto. Recuperado de: http://noticias. uniminuto.edu/uniminuto-en-medios/-/asset_publisher/F9bQ6De3lkpk/ content/historia/1003333?inheritRedirect=false.

Vélez, L. (2011). Administradores Financieros: Esenciales para la competitividad de la empresa contemporánea. Medellín: Fundación Universitaria Esumer.

Vives, A. (2013). Empleo y emprendimiento como responsabilidad social de las empresas. Revista Globalización, Competitividad y Gobernabilidad, 7(3), pp. 16-36.

Weinberg, P. (2004). Empleabilidad. Formación profesional, empleo y empleabilidad. Recuperado de http://www.cinterfor.org.uy/public/spanish/ region/ampro/cinterfor/publ/sala/weinberg/index.htm. 


$$
8
$$

